\title{
Physiopathologie du diabète non insulinodépendant
}

Le diabète non insulinodépendant (DNID) est une maladie fréquente (de 2 à $5 \%$ de la population européenne), mais très hétérogène. L'absence de phénomènes immunologiques et le caractère non obligatoire de l'insulinothérapie pour le pronostic vital à court terme le différencient du diabète insulinodépendant. Le DNID associe une insulinorésistance du métabolisme glucidique hépatique et périphérique et un déficit qualitatif et quantitatif de la sécrétion d'insuline, déficits dont l'importance respective diffère $d$ 'un patient à l'autre et au cours du temps chez un même patient. Le DNID présente une forte composante génétique bien qu'à l'heure actuelle aucun "gène candidat " lié à l'affection n'ait pu être identifié avec certitude.

\section{Philippe Chanson Pascal Ferré José Timsit}

\section{ADRESSES}

P. Chanson : praticien hospilalier universitaire. Service de médecine interne - endocrinologie nutrition, hôpital Lariboisière, 2, rue Ambroise-Parć, 75475 Paris Cedex 10, France.

P. Ferré : direcleur de recherche. Cnrs, centre de recherche sur la nutrition, 9, rue JulesHetzel, 92190 Meudon-Bellevuc, France.

J. Timsit : ancien chef de clinique assistant. Service d'immunologie clinique, hôpital Necker, 161, rue de Sèvres, 75743 Paris e diabète sucré représente un groupe hétérogène de maladies ayant en commun un certain nombre de caractéristiques : élévation chronique de la glycémie et risque accru de développer à long terme des complications vasculaires.

Le diabète sucré, défini selon les critères de l'OMS (Tableau I) a fait l'objet, en 1980, d'une classification étiologique distinguant "type 1 " (le terme "type 1 " appliqué au diabète implique la mise en évidence de phénomènes immunologiques et de marqueurs génétiques) et " type 2 ". En 1985, une classification révisée, plus clinique, a finalement été adoptée, préférant les termes de diabète insulinodépendant (DID) et de diabète non insulinodépendant (DNID) [1].
En dehors des diabètes secondaires (malnutrition, diabète tropical, atteinte pancréatique, anomalies hormonales telles que l'hyper-corticisme ou l'acromégalie, altérations structurales de l'insuline et de son réceptcur...), le DNID se définit par défaut, du fait de l'absence de marqueurs étiologiques spécifiques. Le diabète est dit insulinodépendant si ses symptômes (soif, polyuric, coma...) s'associent à une hyperglycémic et à une cétose : l'administration d'insuline est alors vitale; dans tous les autres cas, même si la persistance de l'hyperglycémie nécessite secondairement l'administration d'insuline, le diabète est considéré comme non insulinodépendant. Le DNID est un problème majeur de santé publique. En Europe, 2 à $5 \%$ 
de la population sont atteints de diabète : il s'agit neuf fois sur dix de DNID, qui réduit l'espérance de vic de 5 à 10 ans en moyenne. Aux États-Unis, en 1986, le coût annuel de la maladie approchait 20 milliards de dollars [2].

L'une des principales difficultés rencontrées dans l'analyse de la physiopathologie du DNID tient à l'hétérogénéité de la maladie. Age de survenue, prévalence en fonction de l'ethnie, degré d'obésité (50 à $80 \%$ des DNID sont obèses), sévérité de l'hyperglycémie, concentration d'insuline circulante, sont extrêmement variables [3]. Le DNID survient généralement après 40 ans, chez des patients présentant volontiers des antécédents familiaux de diabète ct le plus souvent obèses. Certains sousgroupes de patients DNID, possédant des caractéristiques particulières, ont également été décrits. Le MODY (maturity onset diabetes of the young) est un DNID de transmission autosomale dominante, débutant chez le sujet jeune (pic d'incidence à 15-20 ans) [4]. Chez les jeunes Noirs américains, une forme atypique a également été décrite : son début aigu évoque un DID, mais aucun des marqueurs habituels du diabète de type 1 n'est présent et l'insulinothérapic initialement nécessaire peut être interrompuc. Là encore, une transmission autosomale dominante paraît impliquée [5].

En fait, la scule caractéristique commune à tous les DNID est la conjonction d'une insulinorésistance et d'une anomalie de l'insulinosécrétion. Néanmoins, l'importance relative de ces deux facteurs varie considérablement d'un patient à l'autre ou chez un patient donné au cours de l'évolution de la maladie.

\section{Physiopathologie du DNID}

L'homéostasie glucidique. A l'état post-absorptif, c'est-à-dire lorsque l'intestin ne délivre plus de substrats et en particulier de glucose dans la circulation, la glycémie est le reflet, à chaque instant, de la production hépatique de glucose et de son utilisation par les tissus périphériques dont les principaux sont, d'un point de vue quantitatif, le cerveau et les muscles. La production hépatique de glucose est contrôlée par le rapport insuline/glucagon. L'utilisation de glucose à l'état post-absorptif s'effectue pour 70 à $75 \%$ de façon indépendante de l'insuline, en particulier dans le cerveau.

Lors d'une charge orale de glucose, la production hépatique est diminuée d'un facteur deux sous les effets conjugués de l'hyperglycémie, de l'hyperinsulinisme et de la diminution des concentrations circulantes de glucagon, alors que la synthèse hépatique de glycogène à partir du glucose est augmentéc. Les muscles sont les principaux tissus périphériques impliqués dans l'augmentation de l'utilisation de glucose, sous l'effet de l'hyperinsulinisme. Le devenir principal du glucose capté par les muscles est le stockage sous forme de glycogène et, dans une moindre mesure, l'oxydation.

Chez le diabétique non insulinodépendant, on constate unc élévation de la glycémie basale et une intolérance au glucose dont nous allons maintenant discuter les mécanismes.

Production et utilisation de glucose chez les sujets atteints de DNID. A l'état post-absorptif, on constate, en fonction de la gravité de l'hyperglycémie, soit unc production hépatique normale $(1,20 \mathrm{~g} / \mathrm{l}<$ glycémie < $1,40 \mathrm{~g} / \mathrm{l})$, soit une production hépatique élevéc mais qui peut être inhibéc par des doses importantes d'insuline $(1,40 \mathrm{~g} / \mathrm{l}<$ glycémie $<2,0 \mathrm{~g} / \mathrm{l})$, soit enfin une production hépatique élevéc et totalement résistante à l'action de l'insuline (glycémie > $2,0 \mathrm{gl}$ ) [6]. Dans la mesure où, à l'état basal, l'insulinémie des sujets attcints de DNID faiblement hyperglycémiques est en valeur absolue plus élevée que l'insulinémie des témoins, cela démontre une altération de la régulation de la production de glucose par l'insuline et le glucose. Chez les diabétiques faiblement hyperglycémiques, la vitesse d'utilisation du glucose, égale à la vitesse de production hépatique, est normale. Toutefois, cette utilisation normale de glucose est réaliséc en présence d'une insulinémie et d'une glycémie plus élevéc que chez les témoins, ce qui dénote l'existence d'un défaut d'utilisation du glucose [6].

Après une charge en glucose, l'existence d'une résistance périphérique à l'insuline (et donc d'un point de vue quantitatif, essentiellement musculairc) du métabolisme glucidique chez les sujets atteints de DNID, est clairement mise en évidence si l'on considère que chez certains diabétiques, les concentrations absolucs d'insuline circulante sont comparables voire plus élevées que chez les témoins. La technique du clamp euglycémique hyperinsulinique a permis de démontrer et de quantifier cette résistance hépatique et périphérique à l'insuline chez tous les sujets atteints de DNID étudiés [6].

Transmission du message insulinique. L'insuline doit, pour exercer son activité biologique, se lier à un récepteur membranaire, cette liaison
Tableau I

CRITĖRES DIAGNOSTIQUES DU DIABĖTE SUCRÉ (OMS, 1985)

\begin{tabular}{|l|l|l|}
\hline & Glycémie à jeun* & $\begin{array}{c}\text { Glycémie* } 2 \text { heures après } \\
75 \text { ge glucose par voie orale }\end{array}$ \\
\hline Témoins & G $<7,8 \mathrm{mmol} / \mathrm{l}$ & et $\mathrm{G}<7,8 \mathrm{mmol} / \mathrm{l}$ \\
\hline Intolérant au glucose & $\mathrm{G}<7,8 \mathrm{mmol} / \mathrm{l}$ & et $7,8 \mathrm{mmol} / \mathrm{l}<\mathrm{G}<11,1 \mathrm{mmol} / \mathrm{l}$ \\
\hline Diabétique & $\mathrm{G} \geqslant 7,8 \mathrm{mmol} / \mathrm{l}$ & ou $\mathrm{G} \geqslant 11,1 \mathrm{mmol} / \mathrm{l}$ \\
\hline
\end{tabular}

* Glycémie (G) dosée sur plasma veineux; $1 \mathrm{mmol} / \mathrm{l}=0,18 \mathrm{~g} / \mathrm{l}$. 


\section{RÉFÉRENCES}

1. Word Health Organization. Diabetes mellitus. WHO Techn Rep Ser 1985 ; n 727.

2. Huse DM, Oster G, Killen AR, Lacey MJ, Colditz GA. The economic costs of non-insulin-dependent diabetes mellitus. JAMA 1989 ; 262 : 2708-13.

3. Singh BM, Rutter JD, Fitzgerald MG. The natural history of non-insulindependent diabetes mellitus. Baillières Clin Endocrinol Melab 1988 ; 2 : 343-58.

4. Tattersall RB, Fajans S. A difference between the inheritance of a classical juvenile-onset and maturity onset diabetes of young people. Diabeles $1975 ; 24: 44-53$.

5. Winter WE, Maclaren NK, Riley WJ, Clarke DW, Kappy MS, SpillarRP. Maturity-onset diabetes of the youth in black Americans. N Engl J Med 1987 ; 316 ; 285-91.

6. De Fronzo RA. The triumvirate: $\beta$ cell, muscle, liver. A collusion responsible for NIDDM. Diabetes 1988 ; 37 : 667-87.

7. Olefsky JM, Reaven GM. Insulin binding in diabetes : relationships with plasma insulin levels ans insulin sensitivity. Diabeles $1977 ; 26: 680-8$.

8. Brillon DJ, Freidenberg GR, Henry RR, Olefsky JM. Mechanism of defective insulin-receptor kinase activity in NIDDM. Evidence for two receptor populations. Diabetes 1989 ; 38 : 397-403.

9. Caro JF, Ittoop O, Pories WJ, el al. Studies on the mechanism of insulin resistance in the liver from humans with non-insulindependent diabetes. J Clin Invest 1986; 78 : 249-58.

10. Caro J, Sinha MK, Raju SM, et al. Insulin receptor kinase in human skeletal muscle from obese subjects with and without non-insulin-dependent diabetes. $J$ Clin Invest étant suivie de l'émission d'un message intracellulaire. Une légère diminution du nombre des récepteurs de l'insuline dans les tissus des sujets atteints de DNID, sans altération de leur affinité pour l'insuline, a été montrée surtout dans les formes les moins sévères du diabète. Toutefois, cette diminution n'est pas retrouvée chez tous les diabétiques et n'a pu être corrélée à la sévérité de l'atteinte diabétique [7]. En outre, plusieurs équipes ont mis en évidence l'existence d'une altération, en réponse à l'insuline, de la stimulation de l'activité de la tyrosine kinase de la sous-unité $\beta$ du récepteur dans le tissu adipeux [8], le foie [9] et les muscles [10] du sujet atteint de DNID. Un traitement insulinique qui normalise la glycémie entraîne une amélioration notable, voire une disparition, du déficit de l'activité tyrosine kinase du récepteur de l'insuline, ce qui suggère qu'il ne s'agit pas d'un déficit primaire mais d'un déficit secondaire lié à l'altération de l'environnement hormonal et métabolique.

Toutefois, on ne peut éliminer l'hypothèse selon laquelle des défauts génétiques du récepteur de l'insuline puissent être à l'origine de la résistance à l'insuline de certains diabétiques [11].

Résistance hépatique à l'insuline et au glucose. L'importance de la production de glucose dans l'hyperglycémie des sujets atteints de DNID est évidente lorsque l'on établit une corrélation entre hyperglycémie en phase post-absorptive et production hépatique [6]. L'augmentation de la production hépatique de glucose des sujets atteints de DNID apparaît due à une augmentation du flux de la gluconéogenèse, la glycogénolyse restant normale.

Les effecteurs intracellulaires hépatiques du métabolisme glucidique dépendants de l'insuline sont résumés dans le Tableau II. Schématiquement, l'activité et/ou la synthèse d'un certain nombre d'enzymes permettant l'utilisation de glucose par le foie (glycolyse et synthèse du glycogène) sont stimulées par l'insuline et inhibées par le glucagon, alors que la régulation est inverse pour les enzymes permettant la production hépatique de glucose (gluconéogenèse, glycogénolyse).
La première enzyme de la voie glycolytique hépatique, la glucokinase, est particulièrement intéressante car elle n'est présente que dans deux tissus qui ont en charge le maintien de l'homéostasie glucidique, le foie et la cellule $\beta$ du pancréas. La glucokinase est une hexokinase particulière, dont l'affinité pour le substrat est faible ( $\mathrm{Km}$ élevé) et qui, contrairement aux hexokinases des autres tissus, n'est pas inhibée par le produit de sa réaction, le glucose-6-phosphate. Ces particularités lui permettent de traduire de façon quasi linéaire une augmentation de la concentration intracellulaire de glucose par une augmentation de la phosphorylation de ce substrat (le transport du glucose dans l'hépatocyte est assuré par un système à forte capacité, GLUT 2 dans la nomenclature actuelle, qui permet un équilibre rapide entre les concentrations extra- et intracellulaires). Le glucose-6-phosphate ainsi produit peut alors activer la synthèse de glycogène et la glycolyse par effet de masse ou par des mécanismes allostériques. Une diminution de l'activité de la glucokinase entraînera donc une "cécité " hépatique au glucose.

Lorsqu'il existe une carence absolue en insuline, il est facile d'expliquer une augmentation de la production de glucose (Tableau II). Lorsque les concentrations absolues d'insuline sont normales, et en dehors des problèmes liés aux récepteurs de l'insuline, l'existence d'une hyperglucagonémie tout au long de la journée chez le sujet atteint de DNID pourrait, en s'opposant aux effets de l'insuline, contribuer à l'augmentation de la production de glucose. Par ailleurs, chez les sujets atteints de DNID, des concentrations anormalement élevées d'acides gras ont été mises en évidence, et l'oxydation hépatique accrue des acides gras pourrait stimuler la gluconéogenèse en fournissant l'énergie et les cofacteurs nécessaires à son fonctionnement.

Résistance périphérique à l'insuline. Bien que le tissu adipeux ne contribue que modestement à l'utilisation du glucose par rapport au tissu musculaire, il est, d'une part, facilement accessible pour des études in 


\begin{tabular}{|c|} 
TableaU \\
EITAPES MÉTABOLIQUES INTERVENANT \\
DANS L'HOMÉOSTASIE GLUCIDIQUE \\
CONTROLÉES PAR LES HORMONES PANCRÉATIQUES, \\
INSULINE ET GLUCAGON
\end{tabular}

\section{FOIE}

Enzymes de la glycolyse et de la synthèse de glycogène (utilisation de glucose)

- Glucokinase : synthèse stimulée par l'insuline, inhibée par le glucagon

- 6-Phosphofructo-1-kinase : activité stimulée par l'insuline, inhibée par le glucagon

- Pyruvate kinase : activité et synthèse stimulée par l'insuline, inhibées par le glucagon

- Glycogène synthase : activité stimulée par l'insuline, inhibée par le glucagon

\section{Enzymes de la gluconéogenèse et de la glycogénolyse (production de} glucose)

- Fuctose-1,6-bisphosphatase : activité inhibée par l'insuline, stimulée par le glucagon

- Phosphénolpyruvate carboxykinase : synthèse inhibée par l'insuline, stimulée par le glucagon

- Glycogène phosphorylase : activité inhibée par l'insuline, stimulée par le glucagon

\section{Conséquences d'un déficit insulinique (sécrétion ou transmission du} message)

- Augmentation de la production et diminution de l'utilisation de glucose

\section{MUSCLES}

- Transport de glucose : activité stimulée par l'insuline (translocation et activation de transporteurs spécifiques)

- Glycogène synthase : activité stimulée par l'insuline

- Pyruvate déshydrogénase (oxydation du glucose) : activité stimulée par I'insuline

Conséquences d'un déficit insulinique (sécrétion ou transmission du message)

- Diminution de I'utilisation et de l'oxydation du glucose

\section{TISSU ADIPEUX}

\section{Utilisation du glucose et synthèse de lipides}

- Transport de glucose : activité stimulée par l'insuline (translocation et activation de transporteurs spécifiques), synthèse du transporteur stimulée par l'insuline

- Pyruvate déshydrogénase : activité stimulée par l'insuline

- Acétyl-CoA carboxylase : activité et synthèse stimulées par l'insuline

- Synthase des acides gras: synthèse stimulée par l'insuline

\section{Production d'acides gras}

- Triglycéride lipase hormono-sensible : activité inhibée par l'insuline

\section{Conséquences d'un déficit insulinique (sécrétion ou transmission du} message)

- Diminution de l'utilisation de glucose

- Augmentation de la production d'acides gras. Leur utilisation musculaire accrue participe à la diminution de l'oxydation et de l'utilisation du glucose dans les muscles. Par ailleurs, leur oxydation hépatique stimule la production de glucose vitro chez l'homme et, d'autre part, il est impliqué dans la libération des acides gras dont le rôle aggravant dans le maintien de l'hyperglycémie est fortement suspecté. Le tissu adipeux des sujets atteints de DNID est résistant à l'action de l'insuline en ce qui concerne le transport et le métabolisme du glucose [12]. Dans l'adipocyte, l'insuline stimule à court terme le métabolisme glucidique en augmentant la capacité maximale du transport de glucose et en activant les enzymes clés de la lipogenèse (Tableau II). La stimulation du transport de glucose est liée à une translocation vers la membrane plasmique de transporteurs de glucose (spécifiques du muscle et du tissu adipeux et appelés GLUT 4), localisés dans des membranes intracellulaires. Dans les adipocytes de sujets atteints de DNID, le phénomène de translocation de GLUT 4 semble normal ; toutefois le nombre des transporteurs de glucose intracellulaires est réduit [13]. Des études récentes suggèrent que, dans l'adipocyte, l'expression du transporteur de glucose est sous la dépendance de l'insuline [14]. Un déficit insulinique ou une altération de la transmission du message, diminuant l'expression du transporteur de glucose, auront donc comme conséquence d'entraîner une résistance à l'action immédiate de l'hormone (voir également le paragraphe sur la glucotoxicité). Les études concernant les enzymes de la lipogenèse chez le sujet atteint de DNID sont trop rares pour permettre de conclure à un défaut d'activation par l'insuline.

Les concentrations circulantes d'acides gras, indice de lipolyse, sont anormalement élevées chez les sujets atteints de DNID, même non obèses, que ce soit à l'état post-absorptif (malgré l'hyperinsulinisme basal) ou lors de clamps euglycémiques hyperinsuliniques. Ce fait indique que l'insulinorésistance s'étend à la voie métabolique lipolytique [6].

Chez les sujets atteints de DNID, les muscles sont résistants à l'action de l'insuline [6] aussi bien en ce qui concerne le stockage que l'oxydation du glucose, ces anomalies pouvant être localisées au cours de trois étapes : le transport membranaire de glucose, la glycogène synthase, la pyruvate déshydrogénase. 
Dans le muscle, le transporteur de glucose majoritaire est le même que celui du tissu adipeux (GLUT 4) et l'insuline semble là encore agir par translocation du transporteur vers la membrane plasmique (Tableau II). Chez l'homme, les résultats sont actuellement contradictoires en ce qui concerne l'expression de GLUT 4 dans le DNID puisque celle-ci a été trouvée normale dans certaines études et diminuée dans d'autres.

Chez l'homme non diabétique, la capacité de l'insuline à stimuler le captage musculaire de glucose est proportionnclle à l'activation de la glycogène synthase (Tableau II)[15]. Cette activation est, pour une raison qui reste à éclaircir, anormale dans le DNID et, de façon plus générale, chez les sujets insulinorésistants [15]. L'oxydation musculaire du glucose est contrôléc par la pyruvate déshydrogénase, une enzyme mitochondriale dont l'activité est diminuée par des concentrations élevées des produits dé l'oxydation mitochondriale des acides gras. Chez le sujet atteint de DNID, l'augmentation de l'oxydation des acides gras pourrait donc entraîner une diminution de l'oxydation du glucose (Tableau II).

L'intérêt récent concernant l'amyline (voir le paragraphe sur l'insulinosécrétion) a également trouvé un écho dans l'étude de la résistance périphérique à l'insuline. Chez l'animal, l'amyline peut entraîner une diminution marquée des effets périphériques de l'insuline [16]. Toutefois, les concentrations circulantes dans le DNID, de l'ordre de 2 à 4 pM, sont très faibles par rapport aux concentrations efficaces (de l'ordre du nM) permettant de s'opposer aux effets de l'insuline. En outre, il n'existe pas de différence marquée dans les concentrations circulantes d'amyline entre des sujets témoins et des sujets atteints de DNID, que ce soit à l'état basal ou à la suite d'un repas glucidique [17]. La résistance à l'insuline chez les sujets atteints de DNID ne peut expliquer à elle seule l'apparition du diabète. En effet, il existe un certain nombre de situations où l'on trouve une insulinorésistance comparable (obésité) ou beaucoup plus marquée (anomalie génétique du réceptcur de l'insuline) sans hyperglycémie postabsorptive. C'est donc la combinaison d'une insulinorésistance et d'un déficit de sécrétion d'insuline qui détermine l'apparition du DNID.

Insulinosécrétion. Des anomalics de l'insulinosécrétion sont toujours observées au cours du DNID. Elles portent tant sur des aspects quantitatifs (appréciés par l'insulinémie basale ou en réponse à divers stimuli physiologiques de la sécrétion d'insuline) que qualitatifs (disparition de la phase précoce de l'insulinosécrétion après administration intraveineuse de glucose, altération de la potentialisation par le glucose des effets d'autres sécrétagogues, altération de la pulsatilité de l'insulinosécrétion). L'interprétation des études de l'insulinosécrétion au cours du DNID se heurte cependant à plusieurs difficultés : d'une part, la comparaison des résultats publiés dans la littérature doit prendre en compte le degré d'obésité des patients étudiés et la sévérité du trouble glycémique au moment de l'étude (intolérance au glucose, hyperglycémic modérée ou marquée) ; d'autre part, les concentrations d'insuline dans le sang périphérique (paramètre utilisé dans l'immense majorité des études " classiques ") ne reflètent probablement qu'imparfaitement la réalité de l'insulinosécrétion au cours du diabète ; il a en effet été suggéré que l'extraction hépatique de l'insuline portale pourrait être diminuée chez le diabétique. De plus, dans la plupart des cas, les méthodes de dosage immunologique de l'insuline reconnaissent également la pro-insuline (cross-réactivité des anticorps), et il a été montré que le rapport pro-insuline/insuline est plus élevé chez le sujet atteint de DNID que chez le sujet normal. Cependant, ces réserves n'affaiblissent pas, au contraire, la réalité d'une perturbation de l'insulinosécrétion au cours du DNID.

L'insulinémic à jeun (ou "basale ") des sujets atteints de DNID est supérieure, égale ou inférieure à celle de sujets normaux. De fait, lorsqu'on représente l'insulinémie basale en fonction de la glycémie chez des sujets de poids normal et ayant une tolérance au glucose normale ou altérée à des degrés de sévérité croissante, on obtient une courbe en cloche dont le sommet correspond à une insulin secretion in non-insulin-dependent diabetes mellitus. Diabeles Care 1984; 7 491-502. 
glycémie de l'ordre de 1,40 g/l. Audelà de cette valeur, la courbe s'infléchit et l'insulinémie basale s'abaisse, de façon franche, chez les individus les plus hyperglycémiques [6]. Des résultats très voisins sont observés lorsque l'on considère les insulinémies mesurées au cours d'une hyperglycémie provoquée par voie orale (figure 1) [6]. L'élévation de la glycémie de sujets normaux à des valeurs identiques à celles de sujets atteints de DNID d'âge et de poids comparables, élève l'insulinémie au-dessus des valeurs observées chez ces diabétiques. Rapportée à la glycémie ambiante, l'insulinémie basale est ainsi diminuée chez les sujets hyperglycémiques, ce qui évoque, chez ces patients, la notion d'une insulinosécrétion inappropriée à l'hyperglycémie. La diminution, en valeur absolue, de l'insulinémie apparaît de façon encore plus évidente lorsqu'on tient compte de l'augmentation du rapport pro-insuline/insuline observée chez les sujets hyperglycémiques [18].

Après administration intraveineuse d'un bolus de glucose, l'insulinémie augmente physiologiquement en deux phases, le "pic précoce" observé dans les premières minutes, suivi d'une seconde phase prolongée. La disparition du pic précoce est une anomalie constante au cours du DNID, observée pour des niveaux de glycémie basale à peine supérieurs aux valeurs normales (de l'ordre de $1,20 \mathrm{~g} / \mathrm{l})$. Au cours de la phase tardive, l'insulinosécrétion peut apparaître normale voire exagérée, mais, là encore, elle est inappropriée à l'hyperglycémie. Chez les sujets franchement hyperglycémiques, la sécrétion secondaire est diminuée en valeur absolue [19]. Deux arguments militent contre l'hypothèse selon laquelle la perte du pic précoce pourrait constituer un défaut intrinsèque de l'insulinosécrétion préexistant au diabète: cette anomalie n'est pas observée chez des individus normoglycémiques mais étant ultérieurement atteints de DNID, et le pic précoce peut être restauré, au moins partiellement, après une période de contrôle glycémique strict [18].

La réponse insulinémique à d'autres sécrétagogues est également perturbée au cours du DNID. Si l'insulino-

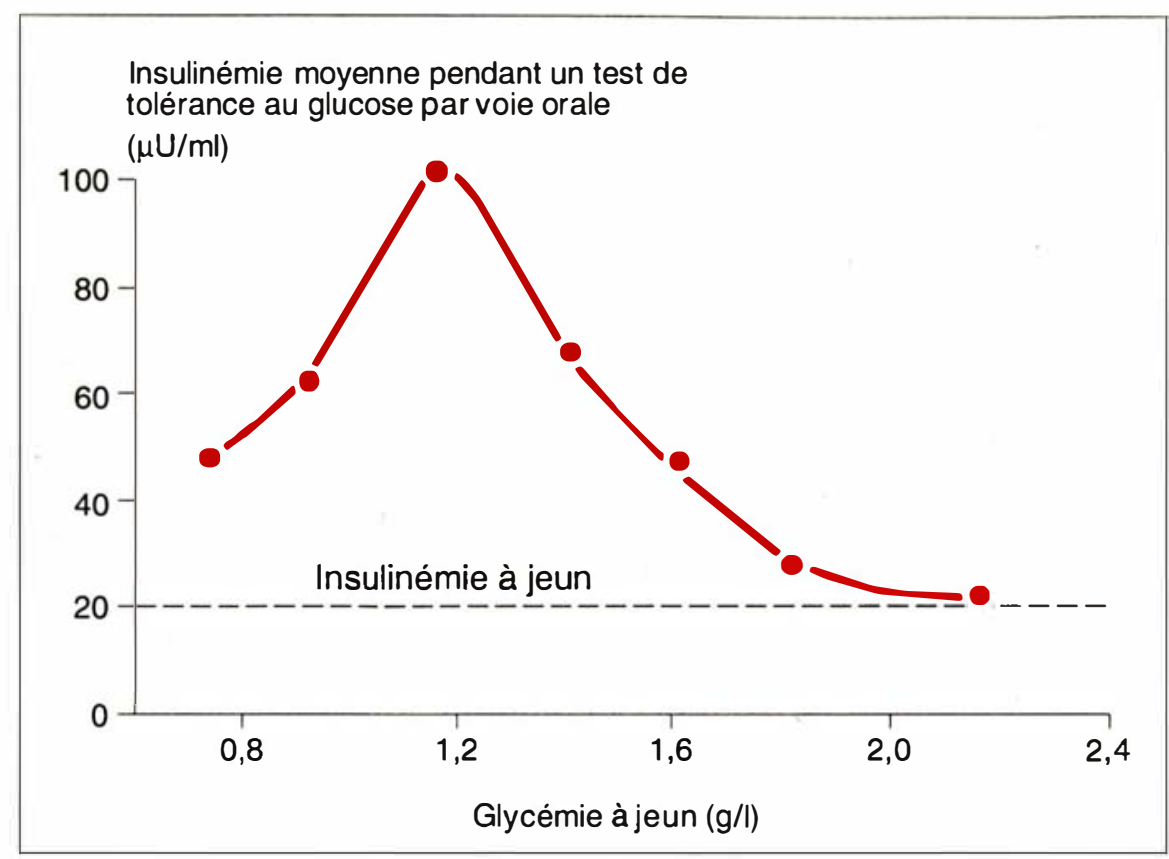

Figure 1. Concentrations moyennes d'insuline obtenues après une charge orale de glucose, chez des sujets présentant un trouble de l'homéostasie glucidique de sévérité croissante. (Adaptée d'après [6].)

sécrétion après injection intraveineuse d'arginine paraît normale, c'est parce qu'elle est potentialisée par l'hyperglycémie. En fait la potentialisation, phénomène phsyiologique, est elle-même altérée. L'augmentation de l'insulinosécrétion en réponse à l'arginine, mesurée à des niveaux croissants de glycémie, est beaucoup plus basse chez le sujet atteint de DNID que chez le sujet normal. De plus, la potentialisation maximale est très diminuée [20]. Ces phénomènes sont d'autant plus marqués que la glycémie basale est élevée mais sont déjà présents pour une hyperglycémie modérée. Les perturbations de l'insulinosécrétion sous divers sécrétagogues peuvent simplement être liées à une anomalie de la potentialisation via une diminution de la "sensibilité " de la cellule $\beta$ au glucose (" cécité " pour le glucose). Il a d'ailleurs été rapporté très récemment une diminution de l'expression du transporteur de glucose (GLUT 2) dans la cellule $\beta$ d'un modèle animal de DNID [21].
L'étude récente des variations ultradiennes de l'insulinémie a mis en évidence des anomalies de la pulsatilité de la sécrétion d'insuline chez les sujets atteints de DNID. Les oscillations rapides se produisant physiologiquement toutes les 10 à 15 minutes sont absentes et l'organisation des pulses suivant les repas est perturbée [22]. Des travaux préliminaires semblent indiquer que cette anomalie pourrait être très précoce dans l'histoire naturelle du DNID, puisqu'elle est observée à un niveau minime d'intolérance au glucose chez des parents au premier degré de sujets atteints de DNID [23].

Chez les sujets atteints de DNID, une diminution de la masse totale des cellules $\beta$ (de l'ordre de 40 à $60 \%$ ), par rapport à des sujets normaux d'âge et de poids comparables, a été mise en évidence par des études combinant histomorphométrie et cytochimie ou immunocytochimie. Cette réduction peut sembler modeste si l'on se réfere aux modèles animaux (notamment le 
glycémie de l'ordre de 1,40 g/l. Audelà de cette valeur, la courbe s'infléchit et l'insulinémie basale s'abaisse, de façon franche, chez les individus les plus hyperglycémiques [6]. Des résultats très voisins sont observés lorsque l'on considère les insulinémies mesurées au cours d'une hyperglycémie provoquée par voie orale (figure 1) [6]. L'élévation de la glycémie de sujets normaux à des valeurs identiques à celles de sujets atteints de DNID d'âge et de poids comparables, élève l'insulinémie au-dessus des valeurs observées chez ces diabétiques. Rapportée à la glycémie ambiante, l'insulinémie basale est ainsi diminuée chez les sujets hyperglycémiques, ce qui évoque, chez ces patients, la notion d'une insulinosécrétion inappropriée à l'hyperglycémie. La diminution, en valeur absolue, de l'insulinémie apparaît de façon encore plus évidente lorsqu'on tient compte de l'augmentation du rapport pro-insuline/insuline observée chez les sujets hyperglycémiques [18].

Après administration intraveineuse d'un bolus de glucose, l'insulinémie augmente physiologiquement en deux phases, le "pic précoce" observé dans les premières minutes, suivi d'une seconde phase prolongée. La disparition du pic précoce est une anomalie constante au cours du DNID, observée pour des niveaux de glycémie basale à peine supérieurs aux valeurs normales (de l'ordre de $1,20 \mathrm{~g} / \mathrm{l})$. Au cours de la phase tardive, l'insulinosécrétion peut apparaître normale voire exagérée, mais, là encore, elle est inappropriée à l'hyperglycémie. Chez les sujets franchement hyperglycémiques, la sécrétion secondaire est diminuée en valeur absolue [19]. Deux arguments militent contre l'hypothèse selon laquelle la perte du pic précoce pourrait constituer un défaut intrinsèque de l'insulinosécrétion préexistant au diabète: cette anomalie n'est pas observée chez des individus normoglycémiques mais étant ultérieurement atteints de DNID, et le pic précoce peut être restauré, au moins partiellement, après une période de contrôle glycémique strict [18].

La réponse insulinémique à d'autres sécrétagogues est également perturbée au cours du DNID. Si l'insulino-

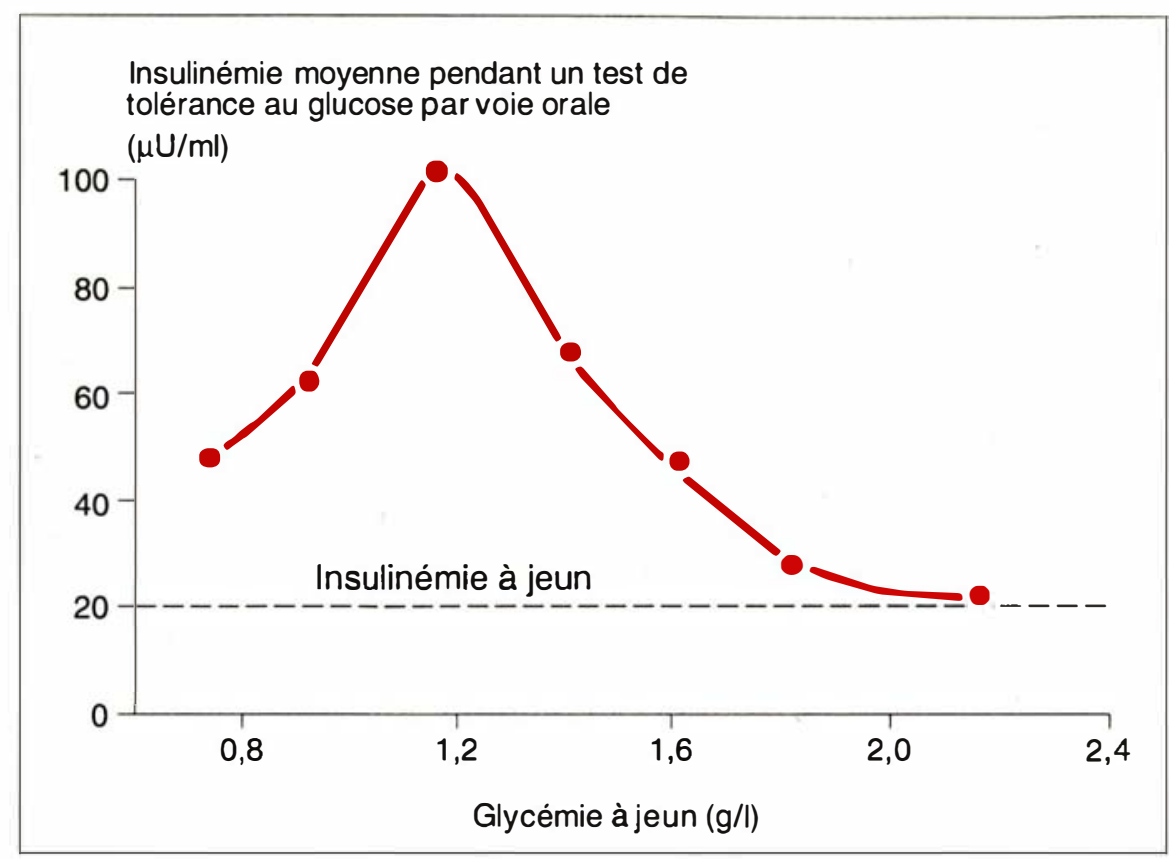

Figure 1. Concentrations moyennes d'insuline obtenues après une charge orale de glucose, chez des sujets présentant un trouble de l'homéostasie glucidique de sévérité croissante. (Adaptée d'après [6].)

sécrétion après injection intraveineuse d'arginine paraît normale, c'est parce qu'elle est potentialisée par l'hyperglycémie. En fait la potentialisation, phénomène phsyiologique, est elle-même altérée. L'augmentation de l'insulinosécrétion en réponse à l'arginine, mesurée à des niveaux croissants de glycémie, est beaucoup plus basse chez le sujet atteint de DNID que chez le sujet normal. De plus, la potentialisation maximale est très diminuée [20]. Ces phénomènes sont d'autant plus marqués que la glycémie basale est élevée mais sont déjà présents pour une hyperglycémie modérée. Les perturbations de l'insulinosécrétion sous divers sécrétagogues peuvent simplement être liées à une anomalie de la potentialisation via une diminution de la "sensibilité " de la cellule $\beta$ au glucose (" cécité " pour le glucose). Il a d'ailleurs été rapporté très récemment une diminution de l'expression du transporteur de glucose (GLUT 2) dans la cellule $\beta$ d'un modèle animal de DNID [21].
L'étude récente des variations ultradiennes de l'insulinémie a mis en évidence des anomalies de la pulsatilité de la sécrétion d'insuline chez les sujets atteints de DNID. Les oscillations rapides se produisant physiologiquement toutes les 10 à 15 minutes sont absentes et l'organisation des pulses suivant les repas est perturbée [22]. Des travaux préliminaires semblent indiquer que cette anomalie pourrait être très précoce dans l'histoire naturelle du DNID, puisqu'elle est observée à un niveau minime d'intolérance au glucose chez des parents au premier degré de sujets atteints de DNID [23].

Chez les sujets atteints de DNID, une diminution de la masse totale des cellules $\beta$ (de l'ordre de 40 à $60 \%$ ), par rapport à des sujets normaux d'âge et de poids comparables, a été mise en évidence par des études combinant histomorphométrie et cytochimie ou immunocytochimie. Cette réduction peut sembler modeste si l'on se réfere aux modèles animaux (notamment le 
21. Johnson JH, Ogawa A, Chen L, et al. Underexpression of $\beta$-cell high $\mathrm{Km}$ transporters in non-insulin-dependent diabetes. Science 1990 ; 250 : 546-9.

22. Polonsky KS, Given BD, Hirsch LJ, et al. Abnormal pattern of insulin secretion in non-insulin-dependent diabetes mellitus. $N$ Engl J Med $1988 ; 318$ : 1231-9.

23. O'Rahilly S, Turner RC, Matthews DR. Impaired pulsatile secretion of insulin in relatives of patients with non-insulindependent diabetes. $N$ Engl J Med 1988 ; $318: 1225-30$.

24. Kendall DM, Sutherland DER, Najarian JS, Goetz FC, Robertson RP. Effects of hemipancreatectomy on insulin secretion and glucose tolerance in healthy humans. $N$ Engl J Med 1990 ; 322 : 898-903.

25. Nishi M, Sanke T, Nagamatsu S, Bell GI, Steiner DF. Islet amyloid polypeptide. A new B cell secretory product related to islet amyloid deposits. J Biol Chem 1990 ; 265 : 4173-6.

26. Nishi M, Bell GI, Steiner DF. Islet amyloid polypeptide (amylin) : no evidence of an abnormal precursor sequence in 25 type 2 (non-insulin-dependent) diabetic patients. Diabetologia $1990 ; 33$ : 628-30.

27. Johnson KH, O'Brien TD, Betsholtz C, Westermark P. Islet amyloid, islet-amyloid polypeptide, and diabetes mellitus. $N$ Engl J Med 1989 ; 321 : 513-8.

28. Clark A, Saad MF, Uren C, Knowler WC, Bennett PH, Turner RC. Islet amyloid polypeptide in diabetic and non-diabetic Pima Indians. Diabetologia 1990 ; 33 : 285-9.

29. Giroix MH, Portha B, Kergoat M, Bailbe D, Picon L. Glucose insensitivity and amino-acid hypersensitivity of insulin release in rats with non-insulin-dependent diabetes : a study with the perfused pancreas. Diabetes $1983: 32$ : 445-51

30. Bonner-Weir S, Trent DF, Weir GC. Partial pancreatectomy in the rat and subsequent defect in glucose-induced insulin release. J Clin Invest 1983 ; 71 : 1544-53.

31. Rossetti L, Laughlin MR. Correction of chronic hyperglycemia with vanadate, but not with phlorizin normalizes in vivo glycogen repletion and in vitro glycogen synthase activity in diabetic skeletal muscle. $J$ Clin Invest $1989 ; 84$ : 892-9.

32. Gauguier D, Bihoreau MT, Ktorza A, Berthoult MS, Picon L. Inheritance of diabetes mellitus as consequence of gestational hyperglycemia. Diabetes $1990 ; 39: 734-9$.

33. Fajans SS. Scope and heterogeneous nature of MODY. Diabetes Care 1990; 13 ;
34. O'Rahilly S, Turner RC. Early-onset type 2 diabetes us. maturity-onset diabetes of youth : evidence for the existence of two discrete diabetic syndromes. Diab Med 1985 ; $5: 224-9$

35. Vinik A, Bell G. Mutant insulin syndromes. Horm Metab Res 1988 ; 20 : 1-10.

36. Taylor SI, Cama A, Kadowaki H, Kadowaki T, Accili D. Mutations of the human insulin receptor gene. Trends Endocr Metab $1990 ; 1$ : 134-9.

37. Matsutani A, Koranyi L, Cox N, Permutt MA. Polymorphisms of GLUT 2 and GLUT 4 genes. Use in evaluation of genetic susceptibility to NIDDM in blacks. Diabetes $1990 ; 39$ : 1534-42.

38. Rotter JJ, Vadheim CM, Rimoin DL. Genetics of diabetes mellitus. In : Rifkin $\mathrm{H}$, Porte D Jr, eds. Ellenberg and Rifkin's Diabetes Mellitus. Theory and Practice. New-York : Elsevier, 1990 : 378-413.

39. Bell GI, Kayano T, Buse JB, et al. Molecular biology of mammalian glucose transporters. Diabetes Care $1990 ; 13$ : 198-208.

40. Li SR, Baroni MG, Oelbaum RS, Stock J. Galton DJ. Association of genetic variant of the glucose transporter with noninsulin-dependent diabetes mellitus. Lancet $1988 ; 1: 368-70$.

41. Turner RC, Matthews DR, Clark A, O'Rahilly S, Rudenski AS, Levy J. Pathogenesis of NIDDM : a disease of deficient insulin secretion. Baillières Clin Endocrinol Metab 1988 ; 2 ; 327-42.

42. Gerich JE. Role of insulin resistance in the pathogenesis of type 2 (non-insulindependent) diabetes mellitus. Bailières Clin Endocrinol Metab 1988 ; 2 : 307-26.

43. O'Rahilly SP, Nugent Z, Rudenski AJ, et al. Beta-cell dysfunction rather than insulin sensitivity is the primary defect in familial type 2 diabetes. Lancet $1986 ; 2$ : 360-3

44. Eriksson J, Franssila-Kallunki S, Ekstrand A, et al. Early metabolic defects in persons at increased risk for non-insulindependent diabetes mellitus. $N$ Engl J Med 1989 : $321: 337-43$

45. Cerasi E, Nesher A. Les défauts de la cellule $\beta$ - langheransienne dans le DNID. In : Rathery $\mathrm{M}$, ed. Journées annuelles de diabétologie de l'Hôtel-Dieu. Paris : Flammarion, 1987: 255-66.

46. Jallut D, Golay A, Munger P, et al. Impaired glucose tolerance and diabetes in obesity : a 6-year follow-up study of glucose metabolism. Metabolism $1990 ; 39$ : 1068-75. rat) dans lesquels une pancréatectomie étendue est nécessaire pour obtenir un diabète. Il faut cependant souligner qu'une pancréatectomie de $50 \%$ s'accompagne d'anomalies de l'insulinosécrétion chez le chien. De plus, il est possible que les cellules $\beta$ restantes soient "pathologiques" dans le DNID. Enfin, une hémipancréatectomie corporéo-caudale induit, chez le sujet sain, une intolérance au glucose ou un diabète dans $25 \%$ des cas. Il faut souligner que chez ces sujets, l'insulinosécrétion mesurée au cours d'une hyperglycémie provoquée par voie orale reste, en valeur absolue, normale [24].

L'anomalie morphologique la plus caractéristique est la présence de dépôts amyloïdes dans les îlots. Ces dépôts sont présents chez les sujets âgés non diabétiques, mais ils sont quantitativement beaucoup plus importants chez les sujets atteints de DNID. Ils sont constitués d'une protéine de 37 acides aminés dont le gène a été cloné, l'amyline (également appelée islet amyloid polypeptide, IAPP). L'amyline est normalement localisée dans les granules de la cellule $\beta$ et cosécrétée avec l'insuline en réponse à une stimulation par le glucose ou l'arginine [25]. La capacité de l'amyline à former des dépôts semble liée à la séquence des acides aminés 25 à 29, mais il ne paraît pas exister d'anomalies des séquences de l'amyline ou de son précurseur chez les sujets atteints de DNID [26]. La localisation des dépôts d'amyline au contact de cellules $\beta$ dont les membranes présentent des lésions ultrastructurales a suggéré que cette protéine pourrait être impliquée dans la physiopathologie du DNID. Le rôle potentiel de ces dépôts sur la diffusion locale du glucose et de l'insuline et/ou un effet auto- ou paracrine de l'amyline sur la sécrétion d'insuline ont été évoqués [27]. Cependant, les données concernant les effets de l'amyline sur la sécrétion et sur les effets périphériques de l'insuline sont encore parcellaires ou contradictoires. De même, la présence de dépôts d'amyline dans le pancréas de singes spontanément diabétiques précède l'intolérance au glucose, mais des résultats opposés ont été obtenus chez les Indiens Pima [28]. Les liens entre la formation de dépôts d'amyline et 
la physiopathologie du DNID (cause ou conséquence?) restent donc incertains.

Le concept de glucotoxicité. Un facteur étiologique qui pourrait être commun à la détérioration de la sécrétion d'insuline et de la sensibilité des tissus est la présence d'une hyperglycémie. En ce qui concerne la sécrétion d'insuline, il a été montré, sur des modèles animaux de diabète expérimental, que la réduction chirurgicale ou chimique de la masse pancréatique, qui entraîne une hyperglycémie chronique, provoquait une altération de la réponse au glucose des cellules $\beta$ restantes $[29,30]$ alors que la réponse aux autres stimuli était peu ou pas affectée. Dans les cas les moins sévères de diabète expérimental, la correction de l'hyperglycémie par la phlorizine, un inhibiteur de la réabsorption tubulaire rénale de glucose (qui provoque donc artificiellement une augmentation de la clairance du glucose), corrigeait également les défauts pancréatiques de sécrétion d'insuline [6]. Toutefois, dans des modèles animaux plus sévères de DNID, l'amélioration de la fonction pancréatique par la phlorizine restait modeste. On peut également noter que, chez les sujets atteints de DNID, la plupart des traitements qui diminuent l'hyperglycémie (régime, traitement par l'insuline ou les sulfonylurées) améliorent la réponse pancréatique des patients au glucose.

La normalisation de la glycémie par la phlorizine, dans les modèles animaux de diabète non insulinodépendant, entraîne également une amélioration de la sensibilité à l'insuline du foie et des tissus périphériques [6], bien que tous les défauts observés chez les animaux diabétiques, en particulier au niveau du stockage de glucose, ne soient pas corrigés [31]. Un certain nombre d'expériences récentes suggèrent que l'hyperglycémie pourrait induire une diminution de l'efficacité du transport du glucose dans les muscles et les adipocytes.

En conclusion, l'hyperglycémie, peutêtre par l'intermédiaire d'une diminution des capacités de transport de glucose, aggrave le DNID en perturbant la sécrétion et l'action de l'insuline, ce qui peut contribuer à l'éta- blissement d'un "cercle vicieux " conduisant à un diabète de plus en plus sévère.

\section{Génétique et DNID}

De nombreux arguments suggèrent que des facteurs génétiques jouent un rôle important dans la survenue du DNID. Ils découlent essentiellement des observations cliniques de cas familiaux, et notamment de jumeaux monozygotes, de la description de formes particulières de DNID dont le MODY (maturity-onset diabetes of the young) constitue un exemple typique, et de l'existence de groupes ethniques à forte incidence de DNID. La part de facteurs non génétiques (mode de vie, alimentation, exercice physique, degré de surcharge pondérale...) dans la modulation de l'expression phénotypique de ces facteurs génétiques doit être prise en compte dans l'interprétation de telles études.

L'observation de jumeaux monozygotes montre que la concordance pour le DNID est très élevée (proche de $100 \%)$ et que dans les paires apparemment discordantes, il existe en fait, chez le sujet non affecté, des anomalies de la tolérance au glucose et de l'insulinosécrétion. Cette concordance est observée même lorsque les jumeaux vivent dans des environnements différents et que leurs poids respectifs sont très différents au moment du diagnostic de DNID. Toutefois, outre l'aspect génétique, ces études pourraient également être expliquées à la lumière de données expérimentales récentes obtenues chez l'animal et montrant qu'un environnement hyperglycémique pendant la vie fotale suffit pour altérer à l'âge adulte la fonction pancréatique [32]. Certains groupes ethniques (Indiens Pima, Hispano-Américains, Nauru) ont une très forte prévalence de DNID, à l'évidence modulée par l'environnement (expression clinique augmentée par l'obésité), et qui est diminuée par le métissage avec des populations à plus faible prévalence (par exemple, $83 \%$ chez les Nauru contre $17 \%$ chez les Nauru métissés). Dans le cas du MODY, un mode de transmission autosomique dominant du DNID peut être évoqué, mais, même dans ce cadre relativement bien défini, il existe des variations dans la pénétrance du (ou des) gène(s) [33]. Dans les études de familles de "diabète de type 2 de survenue précoce ", l'hypothèse d'une transmission de gène(s) en double dose a été proposée pour rendre compte de la forte incidence de diabète ou d'intolérance au glucose observée chez les parents et dans la fratrie [34]. Des arguments épidémiologiques recueillis chez les Pima vont également dans ce sens. Cependant, pour la majorité des cas de DNID, un mode unique de transmission ne peut être décrit (" cauchemar du généticien ").

Des mutations ponctuelles de la partie codante du gène de l'insuline [35] ou du récepteur de l'insuline [36] ont été décrites dans un certain nombre de familles. Il est intéressant de noter que les sujets porteurs de l'anomalie génique n'ont pas tous le même degré d'intolérance au glucose, ce qui suggère, indépendamment des facteurs d'environnement, l'intervention d'autres anomalies. Ces cas sont rares (par exemple, moins de $0,5 \%$ de mutation du gène de l'insuline dans une population de sujets atteints de DNID) et, à l'heure actuelle, pour l'immense majorité des sujets atteints de DNID, aucun gène de susceptibilité n'a été identifié avec certitude. La recherche du, ou peut-être des, gène(s) en cause est rendue difficile par des facteurs tels que l'hétérogénéité de la maladie, la méconnaissance des mécanismes initiateurs du DNID et l'existence d'une variabilité physiologique de l'insulinosécrétion et de la sensibilité à l'insuline, ellemême d'origine en partie génétique. Le polymorphisme de différents "gènes candidats ", impliqués dans les mécanismes de la sécrétion d'insuline ou de ses effets périphériques, a été étudié. L'existence d'un polymorphisme de GLUT 2 (transporteur de glucose présent dans la cellule $\beta$ et dans le foie) a été mis en évidence dans la population noire américaine [37]. Toutefois, il n'a pas été trouvé d'association entre ce polymorphisme et le DNID [37]. Le gène de la glucokinase de la cellule $\beta$ constitue également un candidat important, puisque l'activité de cette enzyme détermine la première étape des mécanismes conduisant à la sécrétion d'insuline. La description d'une asso- 
ciation du DNID avec un polymorphisme de restriction de la région flanquante 5' du gène de l'insuline n'a pas été confirmée.

En ce qui concerne l'action de l'insuline, plusieurs études ont montré l'association d'un polymorphisme du gène du récepteur de l'insuline avec une diminution de la sensibilité à l'insuline et, dans certains cas, avec le DNID [38]. En outre, le clonage des gènes des différents transporteurs membranaires du glucose a ouvert de nouvelles perspectives [39]. Des résultats contradictoires ont été obtenus quant à l'association d'un polymorphisme du gène de GLUT 1 (transporteur constitutif retrouvé dans la plupart des cellules) au DNID [38, 40]. De même, il n'a pas été observé d'association du polymorphisme de GLUT 4 (transporteur insulinosensible du muscle et du tissu adipeux) au DNID [37, 39]. Enfin, comme pour la cellule $\beta$, la glucokinase hépatique pourrait représenter un " gène candidat " intéressant. En conclusion, à l'heure actuelle, aucun " gène candidat " satisfaisant n'a pu être identifié.

\section{Vers une histoire naturelle du DNID?}

Histoire naturelle de la rćsistance à l'insuline et de l'hyperinsulinisme dans le diabète non insulinodćpendant. In : Rathery $M$, ed. Journées annuelles de diabétologie de l'Hôtel-Dieu. Paris: Flammarion, 1990 : 201-8.

50. Hansen BC, Bodkin NL. Heterogeneity of insulin responses: phases leading to type 2 (non-insulin-dependent) diabetes mellitus in the rhesus monkey. Diabetologia 1986 ; 29 : 713-9.

51. Bennett PH, Pettitt DJ, Saad MF, Bogardus C. Lilloja S, Knowler WC. Diabète non insulinodćpendant : ce que nous ont appris les ćtudes réalisćes parmi les Indiens Pima. In : Rathery M, ed. Journées annuelles de diabélologie de l'Hôtel-Dieu. Paris: Flammarion, 1990: 231-45.

52. Saad MF, Knowler IVC, Pettitt DJ, Nelson RG, Mott DM, Bennett PH. The natural history of impaired glucose tolerance in the Pima Indians. $N$ Engl J Med 1988 ; 319 : 1500-6.

53. Lilloja S, Mott DM, Howard BV, et al. Impaired glucose tolerance as a disorder of insulin action. Longitudinal and crosssectional studies in Pima Indians. $N$ Engl $J$ Med 1988 ; 318 : 1217-25.

54. Saad MF, Knowler WC, Pettitt DJ, Nelson RG, Mott DM, Bennett PH. Sequential changes in serum insulin concentration during development of non-insulin-
Il est clairement établi qu'avant de devenir diabétique, le sujet " normal " passe par une phase d'intolérance au glucose. Toutefois, une partie seulement des individus présentant une intolérance au glucose évolue vers un diabète patent [3].

Il est actuellement difficile de déterminer si la lésion primitive à l'origine de la cascade des événements qui conduit au DNID est une insulinorésistance ou un défaut d'insulinosécrétion [41-47]. Quelle qu'en soit la cause, l'hyperglycémie peut majorer l'insulinorésistance et le défaut d'insulinosécrétion, ce qui aggrave encore l'hyperglycémie (voir le concept de glucotoxicité). Deux modes d'entrée dans la maladie sont donc possibles. On peut concevoir qu'une association des deux troubles soit nécessaire pour qu'un diabète survienne ; au niveau de l'insulinosécrétion, on peut envisager un défaut génétique ou acquis de la cellule $\beta$ (vieillissement, par exemple) et de la même façon, des facteurs génétiques et/ou d'environnement (obésité, alimentation, activité physique...) peuvent perturber la sensibilité à l'insuline. Les rôles respectifs de ces deux perturbations pourraient être très variables d'un individu à l'autre, ce qui rendrait compte de l'hétérogénéité clinique du DNID, et pourraient de plus évoluer dans le temps, ce qui expliquerait la variation du trouble glycémique chez un individu donné $[48,49]$. Les données disponibles pour décrire plus précisément la séquence des événements et tenter d'en établir les déterminants proviennent d'études transversales ou longitudinales.

Les études transversales comparent des patients à des niveaux variables de tolérance au glucose, partant du postulat selon lequel chaque groupe représente un stade évolutif du processus pathologique. C'est ainsi que De Fronzo a pu proposer, à partir de l'étude parallèle de l'insulinosécrétion et de la sensibilité à l'insuline, un modèle dans lequel l'absence de compensation du défaut de sensibilité par une insulinosécrétion adéquate fait basculer le patient vers le diabète avéré [6] (figure 2).

Les études longitudinales prenant en compte l'ensemble du processus évolutif sont peu nombreuses. Elles souffrent de deux défauts majeurs : elles concernent des populations dans lesquelles la prévalence de la maladie est élevée et/ou dont le diabète est associé à l'obésité. Dans un modèle animal de singes obèses suivis pendant six années, une évolution comparable à celle proposée par les études transversales a été décrite [50]. Chez les Indiens Pima [51], chez des sujets obèses [46] et chez des enfants dont les deux parents étaient diabétiques [47], des conclusions tout à fait similaires ont pu être tirées : le défaut de sensibilité à l'insuline est présent dans les phases précoces [46, 47, 52, 53 ] et le diabète patent est associé à une diminution de l'insulinosécrétion $[46,54]$. Même si le schéma proposé est séduisant, il n'est pas possible, à l'heure actuelle, d'extrapoler ces données à l'ensemble des diabétiques non insulinodépendants

\section{Remerciements}

Nous remercions le Dr Errol Cerasi pour ses conseils lors de la rédaction de cet article. 


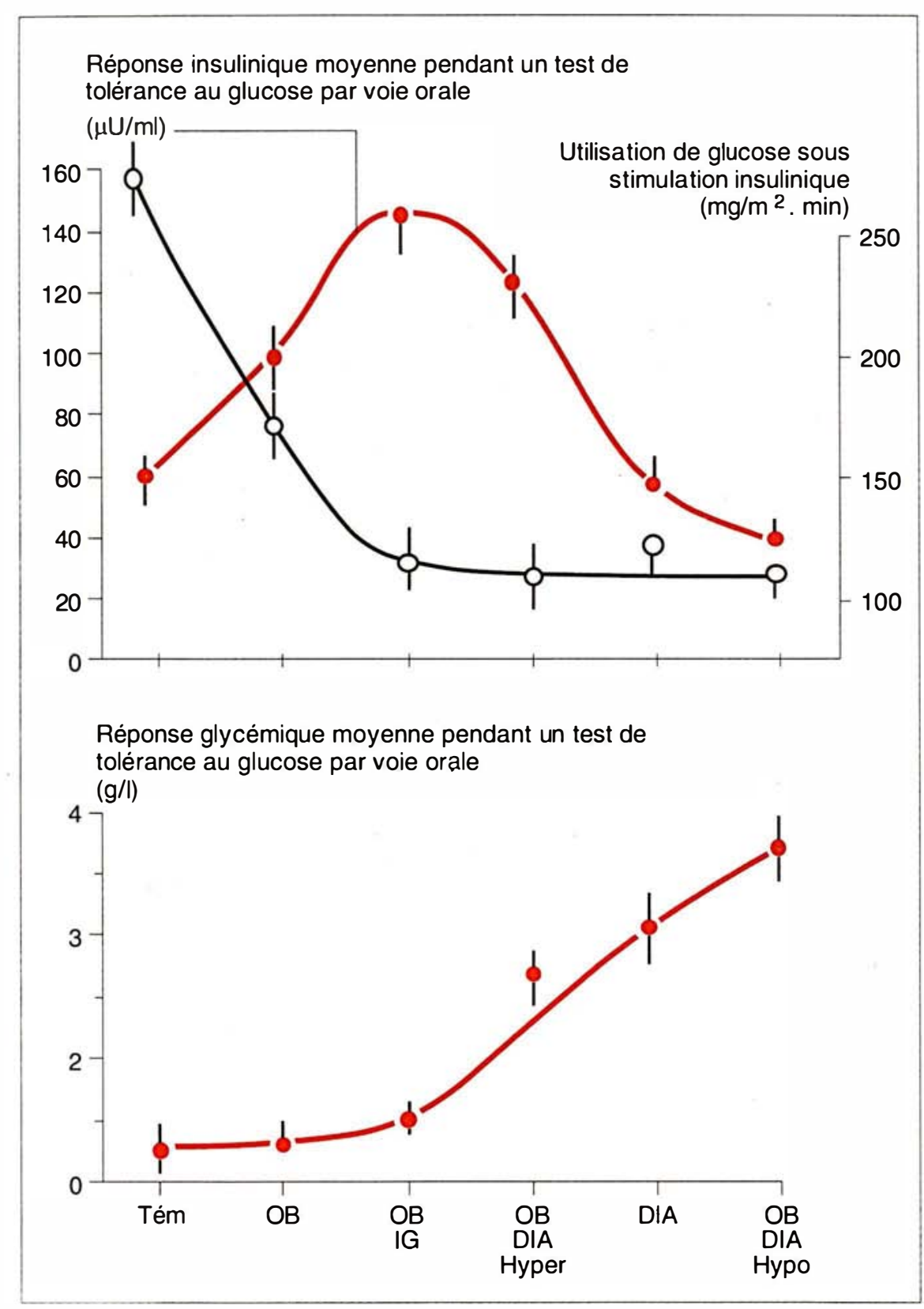

Figure 2. Glycémie et insulinémie au cours d'un test de tolérance au glucose par voie orale. Corrélation avec la sensibilité à l'insuline. La glycémie et l'insulinémie ont été mesurées au cours d'un test de tolérance au glucose (100 g par voie orale) chez des sujets témoins (Tém), obèses non diabétiques $(O B)$, obèses intolérants au glucose $(O B(G)$, obèses diabétiques hyperinsulinémiques (OB DIA HYPER), diabétiques de poids normal (DIA), obèses diabétiques hypo-insulinémiques (OB DIA HYPO). La sensibilité tissulaire a été appréciée au cours d'un "clamp " euglycémique hyperinsulinémique : l'insulinémie est augmentée grâce à une perfusion continue d'insuline et la glycémie est maintenue à une valeur fixe grâce à une perfusion ajustable de glucose. Le glucose qui doit être perfusé pour maintenir la glycémie constante est alors le reflet de la sensibilité tissulaire à l'insuline. (Adaptée d'après [6].)

$m / s n^{\circ}$ 4. wol. 7, arril gl

\section{Summary}

Pathogenesis of non-insulindependent diabetes mellitus

Non-insulin-dependent diabetes mellitus (NIDDM) is a frequent disease (2-5\% of the European population) characterized by a chronic hyperglycemia. NIDDM can be differentiated from the insulin-dependent diabetes by the absence of immunological phenomenon and the fact that insulin therapy is not necessary for the survival in the short-term range. NIDDM is extremely heterogeneous in term of severity of the disease and patient characteristics. NIDDM is concomitant with an insulin resistance of hepatic and peripheral glucose metabolism and with a qualitatively and quantitatively deficient insulin secretion. The respective importance of these two factors in the etiology of NIDDM varies among patients as well as in the same patient at different periods of time. The evidence suggests that NIDDM is a genetic disorder although no candidate gene has been presently clearly identified for either the insulin secretion or insulin action sides.

\section{TIRÉS A PART}

P. Ferré. 